\title{
L'écriture francophone et la question foncière : \\ L’exemple des géocultures kanak et guin-ewe
}

\author{
Laté Lawson-Hellu \\ University of Western Ontario
}

\begin{abstract}
Résumé
La question foncière, pour les écritures francophones, intègre les problématiques juridiques de la modernité pour une telle question, mais aussi les incidences de l'histoire coloniale sur les cultures et espaces humains concernés par une telle question. Dans les deux exemples retenus de la « géoculture » kanak et de la «géoculture » guin-ewe, dans l'océan Pacifique et en Afrique subsaharienne, le paradigme proposé de la «question de la terre » permet de subsumer une telle relation sociosémiotique entre l'écriture et sa réalité socio-culturelle et discursive de référence. Pour les deux espaces culturels, le paradigme épistémologique de la géoculture proposé permet également d'indiquer dans quelles mesures, dès lors, l'œuvre de l'écrivain francophone, tel Félix Couchoro, problématise l'Histoire en y inscrivant sa subjectivité en termes de résistance.
\end{abstract}

Mots-clés : Culture kanak, Culture guin-ewe, Écriture francophone, Géoculture, Identité, Terre et territoire, Histoire coloniale, Félix Couchoro, Déwé Gorodé. 


\section{Introduction}

Le droit, positif ou coutumier, même dans le contexte francophone, où il se rencontre sous les deux modalités juridiques, pose la relation de propriété ou non entre l'individu, sa collectivité et la terre supposée lui procurer les moyens de sa subsistance ou de sa vie. La question coloniale propre au fait francophone y ajoute la question de la modernité qui redéfinit une telle relation au point d'en faire la matière du discours littéraire produit par l'individu écrivain « francophone », c'est-à-dire à partir de l'incidence fondamentale de l'histoire sur ce qui constitue alors sa terre. Dans la Charte du Peuple Kanak promulguée, par exemple, en 2014 sur les «valeurs et principes fondamentaux de la civilisation Kanak » (Sénat Coutumier de la Nouvelle-Calédonie, 2014 : 4), le rôle déstabilisateur de l'histoire coloniale dans l'intégrité d'ensemble du peuple est ainsi précisé, qui rappelle aussi la nécessité de démarches concertées, telle celle de la Charte, comme dans le cadre du processus de décolonisation entamée par le peuple kanak, en Nouvelle-Calédonie, contre l'histoire coloniale depuis au moins les années 1950, et, sur le plan politique, les années 1980 :

Tout comme dans la plupart des régions de l'Océanie, l'histoire première des clans Kanak et de leur déplacement dans l'espace a été totalement bouleversée par la colonisation et par l'arrivée de la religion au milieu du XIX $^{\mathrm{e}}$ siècle. La prise de possession de la Nouvelle-Calédonie par la France proclamée à Balade le 24 septembre 1853 sera pour le Peuple Kanak une nouvelle étape de son destin.

La colonisation va ériger les nouvelles frontières de cette colonie française des antipodes dont la population sera désormais juridiquement et artificiellement séparée du reste du monde mélanésien. [...] 
La création des réserves lors de l'indigénat a été un instrument de ségrégation et de contrôle des Kanak tout en favorisant l'accaparement des terres pour la colonisation.

Définir le rapport entre la production littéraire ou artistique culturelle francophone et la question foncière constitue ainsi une entreprise à la fois épistémologique et herméneutique où interviennent les conditions générales de production, de signification et d'intelligibilité holistique des productions culturelles ainsi considérées.

Pour le champ francophone, l'un des aspects retenus, qui permet une telle mise au jour socio-sémiotique, demeure donc la question de la terre. Celle-ci recouvre à la fois la problématique immédiatement foncière à laquelle renvoie une telle question ; elle recouvre aussi les propres conditions épistémologiques d'appréhension de telle question, que ce soit du point de vue historique, culturel, politique, économique, juridique, social, géographique ou humain, tel que l'individu écrivant y prend position dans la production de son œuvre. Dans le cas francophone, la question de la terre, dans sa première acception, renvoie naturellement à toutes les problématiques foncières qui s'élèvent jusqu'à la dimension métaphysique ou ontologique où l'individu se comprend dans son inscription dans un territoire. Ici, ce peut être le pays, la culture, la tribu, l'ethnie, la région, etc., où, pour la question littéraire, une telle inscription informe le discours identitaire que produit l'individu sur son appartenance ou non à ce « territoire » ainsi qu'à la dimension collective de cette appartenance ou non. Dans son deuxième aspect, plus vaste, intervient la perspective discursive axiologique, normative, évaluative ou spéculative que l'individu produit sur sa terre d'appartenance ou non, dans les termes du rapport de cette terre à l'histoire, à la politique, au fait économique, au fait idéologique, au fait humain et au fait 
environnementaliste, comme désormais à partir des premières décennies du XIX ${ }^{\mathrm{e}}$ siècle. La Charte du Peuple Kanak (2014 : 4-5) fait mention de ce rapport à l'Histoire, dans l'exemple ponctuel de la Nouvelle-Calédonie et de la démarche de résistance apposée alors à l'histoire déstabilisatrice :

Pour résister à l'entreprise coloniale de spoliation et d'anéantissement, les atouts du peuple Kanak auront été, d'une part, l'autonomie des chefferies entre elles, ce qui leur a permis d'éviter une guerre coloniale frontale et, d'autre part, la capacité de la Civilisation Kanak à s'adapter en s'appuyant sur des valeurs sociétales sûres. Ces valeurs qui fondent encore aujourd'hui l'organisation sociale Kanak, sont l'hospitalité, la générosité, le respect à tous les niveaux, la dignité, le travail, encadrées par la force des relations et de l'organisation sociale de la chefferie. Elles ont porté une dynamique interne forte, laquelle a permis de s'adapter et d'intégrer les nouveaux arrivants.

$\mathrm{Si}$ une telle perspective discursive et normative recoupe ainsi avec les aspects immatériels de la terre ou du territoire d'inscription et de constitution discursive, c'est-à-dire en termes d'appartenance identitaire, c'est à travers toute la problématique de la culture que se définira également le discours identitaire produit par l'individu écrivant. Dans la question francophone, cette perspective discursive et évaluative se concrétise dans la dualité que l'histoire coloniale aura créée entre un fait de territoire et de culture autochtone et un fait de territoire et de culture allogène généré par le fait colonial. Le discours identitaire ou la posture ontologique de l'individu écrivant francophone traduira l'appréhension épistémologique de cette dualité par l'individu. La question ontologique posée dans la réflexion, ici, s'inscrit donc dans le filtre axiologique que constitue le fait historique colonial. Elle prend en compte à la fois la réalité socio-historique sur le terrain, dans les deux exemples retenus de la terre kanak, en NouvelleCalédonie, et de l'espace guin, au Togo, de même qu'elle prend en compte le 
discours identitaire produit par l'écrivain, l'écrivaine, issus de tels espaces. Le paradigme théorique de la géoculture lui donne ensuite sa configuration épistémologique. À ce titre, il s'établit, par exemple, la corrélation entre le discours sur la terre, tel que produit par l'individu, et la subjectivité ontologique de cet individu-sujet, homme ou femme, inscrit dans le paradigme francophone. En tant que paradigme critique, le discours sur la terre a fait l'objet d'une présentation théorique, épistémologique, dans le cadre, herméneutique, de la définition du texte francophone (Laté Lawson-Hellu, 2018). Il est repris ici dans son sens référentiel identitaire tel qu'il informe ou rend compte du discours de l'individu dans sa subjectivité francophone. Une telle subjectivité, du fait de l'histoire coloniale, problématise la question de la terre, laquelle devient, à la limite, une revendication politique et territoriale.

\section{La question de la terre}

La relation entre le principe du discours sur la terre et l'expression subjective que constitue l'expression littéraire, dans le contexte francophone, trouve son meilleur reflet, il nous semble, dans la question de la terre identifiable dans le contexte historique et politique néo-calédonien du courant du $\mathrm{XX}^{\mathrm{e}}$ siècle. Pour mémoire, la question coloniale qui a conduit à un processus de décolonisation dès les années 1980 dans ce territoire d'outre-mer français, s'inscrit dans un contentieux historique dont il était prévu l'aboutissement dans le courant de l'année 2018 avec la possibilité, pour ce territoire, de sortir de son statut de territoire colonisé. Un tel contentieux repose sur l'occupation, par la France, au milieu du XIX ${ }^{\mathrm{e}}$ siècle, de ce territoire des Kanak, membres des peuples mélanésiens, ou "Noirs», du Pacifique. Les ramifications de ces peuples mélanésiens en termes culturels et humains s'étendent aux îles voisines de la 
région, y compris l'Australie. Pour ce contentieux, des terres auront été confisquées dès le $\mathrm{XIX}^{\mathrm{e}}$ siècle et des zones de réaffectation des peuples autochtones auront été mises sur pied pour déboucher sur des refus traduits, dans certains cas comme en Nouvelle-Calédonie, en revendications formelles d'autonomie ou d'indépendance. Un tel processus, dans ses diverses mutations en Nouvelle-Calédonie, aura abouti, dès les années 1980, à des accords politiques sur la base desquels des revendications nouvelles se sont dégagées qui tournent autour de la nature du «pays » devant émerger du processus de décolonisation entrepris. Dans ces nouvelles revendications, est évoqué le statut du territoire à associer ou non au peuple autochtone, les Kanak, ou, à travers le rattachement à la France, à ceux qui se sont établis par la suite, dans le cadre de la colonisation. Dans ce cadre, des décisions politiques ponctuelles auront visé à modifier la composition démographique du territoire afin de faire barrage à la revendication identitaire autochtone. Joël Dauphiné (1998 : 21-22) évoque à ce titre le boom économique des années 1960-1970 autour de l'exploitation minière du nickel, lequel crée, dans les années 1970, un flux d'immigration européenne et autour des îles du Pacifique. Un tel flux, appuyé par le pouvoir en métropole, aura eu pour résultat de changer l'état démographique de la Nouvelle-Calédonie aux dépens de sa population autochtone :

Avec l'arrivée massive d'au moins 10000 Européens, dont un certain nombre de rapatriés d'Algérie, et la poursuite de l'immigration wallisienne, les Mélanésiens, pourtant démographiquement très dynamiques, deviennent de plus en plus minoritaires dans leur archipel. Perspective qui satisfait manifestement les pouvoirs publics, lesquels n'ont pas hésité à accompagner ce phénomène par des encouragements semi-officiels à « faire du Blanc $»^{14}$.

\footnotetext{
${ }^{14}$ En note : «Tel est le but poursuivi par le Premier ministre Pierre Messmer dont une circulaire de 1972 visait à encourager l'immigration en Nouvelle-Calédonie pour prévenir et si possible
} 
La revendication autochtone, justifiée par la suite dans la reconnaissance, par l'ONU, du statut d'espace colonisé de la Nouvelle-Calédonie ${ }^{15}$, pourtant membre de l'ensemble territorial national français, et par le processus de décolonisation dont elle fait l'objet, se heurte à des revendications parallèles, également, de maintien du territoire dans l'ensemble national français. La question de la terre, susceptible alors d'informer le discours de l'écrivain ou de l'écrivaine issue de ce territoire se trouve fondamentalement impliquée dans un processus à la fois administratif, politique, économique, culturel, discursif identitaire, géographique, humain et géopolitique qui dérive lui-même d'un processus historique colonial bien déterminé. Pour la réflexion épistémologique, l'espace néo-calédonien devient la mesure du processus historique et colonial qui, durant le $\mathrm{XX}^{\mathrm{e}}$ siècle, avait abouti à la création de l'espace francophone officiel. C'était, notamment, à travers la question des revendications nationalistes de l'après-deuxième Guerre mondiale, puis à travers les processus d'indépendances politiques qui donnent naissance aux pays post-coloniaux devant former la majorité des pays francophones réunis par exemple au sein de structures internationales comme celle de la Francophonie. Dans le cas précis de la Nouvelle-Calédonie, l'actualité de cette question, qui semblait pourtant révolue dans le courant du $\mathrm{XX}^{\mathrm{e}}$ siècle, redonne toute sa pertinence à la dimension holistique du rapport de l'individu francophone à sa terre, telle que cette terre est problématisée par le fait colonial. C'est un tel tort de l'histoire coloniale à sa terre que l'individu francophone, au

noyer les éventuelles revendications nationalistes des Canaques. Cette politique était notamment approuvée dans le territoire par le maire de Nouméa, Roger Larocque. »

${ }^{15}$ Il existe effectivement une résolution des Nations-Unies, la résolution 1514, qui reconnaît le droit à l'auto-détermination du Peuple kanak, et, ainsi, à la décolonisation de la NouvelleCalédonie en tant que territoire non autonome. La Nouvelle-Calédonie est inscrite à ce titre sur la liste des Nations-Unies sur les territoires à décoloniser. La Charte du Peuple Kanak en fait mention dans son préambule (Op. cit. : p. 8). 
sens institutionnel, et donc politique et géostratégique, continue de juguler à son niveau individuel et à celui de sa collectivité, avec la permanence précise du fait historique d'antan dont les formes vont des plus implicites, comme celle de la langue, aux formes les plus explicites, comme celle qui fait poser la question de l'indépendance en Nouvelle-Calédonie. D'où la question ontologique.

Les bases de la revendication d'indépendance, pour la Nouvelle-Calédonie, demeurent en effet foncièrement culturelles, en référence aux faits de culture par lesquels se spécifie le territoire, tout comme elles le demeurent aussi culturellement dans les espaces de la francophonie devenus États indépendants, où les écrivains, les écrivaines, en font la matière de leurs écritures. Dans ces espaces, tel en Nouvelle-Calédonie, et jusqu'au début du troisième millénaire de l'ère chrétienne, la question de la terre se formule dans des termes politiques qui convoquent tous les autres aspects de la vie collective propre au modèle de société introduit par la modernité européenne ou occidentale, paupérisation, fragilisation du tissu collectif, crises de l'environnement, etc. La compréhension $\mathrm{du}$ fait francophone passe donc par la prise en compte de la question de la terre pour les subjectivités francophones qui s'expriment dans les productions culturelles, telle la littérature, mais aussi, au bout du compte, par le discours de ces subjectivités francophones, sur leur rapport antagonique ou non à l'incidence de l'histoire sur leurs terres respectives. C'est dans ce contexte, qui prend une dimension épistémologique, que se comprend l'œuvre de l'écrivain Félix Couchoro achevée il y a un peu plus d'un demi-siècle ${ }^{16}$.

Chez Félix Couchoro, la question de la terre est ainsi au cœur de l'intelligibilité socio-discursive de l'écriture et de la réflexion historique qui se

\footnotetext{
${ }^{16}$ Cette œuvre débute en effet à la fin des années 1920 et s'achève à la fin de la décennie 1960 .
} 
prononce, chez lui, sur les conditions de l'histoire coloniale telles qu'apposées au réel vécu ou mis en écriture. Dans ce sens, les deux contextes de la géographie et de la culture permettent de saisir, comme chez lui, à travers le paradigme du discours sur la terre, la pertinence du fait francophone qui s'exprime à travers les subjectivités que forment les voix des écrivains et écrivaines qui s'y inscrivent. Leurs voix s'adossent, en cela, aux spécificités des subjectivités collectives dont elles se réclament ou qu'elles défendent, ou problématisent. Pour Félix Couchoro, ce sera le fait culturel guin-ewe, tout comme, en Nouvelle-Calédonie, le fait culturel kanak. Ce fait culturel justifie le discours de l'individu sur sa terre, son territoire, son pays. Janvier Améla (2001 : 442) rend compte de cet attachement de Félix Couchoro, par-delà son parcours individuel ou personnel, à la ville d'Aneho, capitale du pays Guin et ancienne capitale du Togo colonial ; cette ville devient l'espace « mythique » dans l'ensemble de son œuvre :

De Ouidah à Grand-Popo, puis à Petit-Popo (Aneho) jusqu'à Lomé, Aflao-Lomé, et finalement de nouveau et pour toujours à Ouidah qui abrite sa dernière demeure, la boucle aura été bouclée, et Aneho apparaîtra sur ce tracé existentiel comme une halte essentielle, un havre tranquille et sûr, la station particulière de son reposoir, l'autre Ile-Ifè mythique de la Côte des Esclaves, le lieu d'origine de toutes nos légendes, le foyer de refondation du monde nouveau que le romancier prédit ancré dans l'ancestralité et ouvert sur la modernité comme la cité des Guin-Mina l'a toujours été.

Toute la question du partage colonial des espaces géoculturels de l'Afrique précoloniale informe donc la posture discursive et militante de cet écrivain. En Nouvelle-Calédonie, la contestation de l'occupation coloniale de la terre kanak conduit à la demande d'indépendance jusqu'aux premières décennies du $\mathrm{XXI}^{\mathrm{e}}$ siècle. Dans l'espace guin-ewe, ce sont les mêmes conditions de l'occupation européenne, mais du pays «ewe », et dans la même période coloniale initiale et 
emblématique de la fin du XIX ${ }^{\mathrm{e}}$ siècle, qui expliquent le mouvement nationaliste ewe à partir duquel se définit, sur le plan politique, puis symbolique littéraire, la posture militante de l'écrivain. Pour J. Améla (ibidem.), une telle posture ne démontre pas seulement la valeur tellurique intrinsèque à la représentation de la ville d'Aného, chez l'écrivain, mais bien aussi le rapport de cette culture autochtone à l'histoire coloniale. L'écrivain, dans cette posture discursive, donne la leçon, pour lui, de la prévalence de l'espace rural, dans un sens à la fois politique et épistémologique que symbolise alors la ville historique mise en écriture :

L'œuvre de Couchoro s'est développé[e] en dehors du courant de prescription, de proscription et de prohibition de la négritude, et s'est intéressé[e] plutôt aux réalités autochtones que l'écrivain s'est bien gardé de percevoir dans les yeux de l'homme blanc. Cette option est éminemment idéologique et tend à montrer que les cultures autochtones ont leur propre régulation, qu'elles ont toujours existé sans référence aux "oreilles rouges", et que, contrairement à ce qu'on a pu dire, à Aneho au moins, une culture africaine ancrée dans l'ancestralité est cependant restée tournée vers la modernité sans que la colonisation "y ait été pour quelque chose".

$\mathrm{Si}$, dans l'espace kanak, il est question d'un discours indépendantiste, dans celui de Félix Couchoro, il s'agit d'un discours nationaliste qui conduit à l'indépendance du Togo telle qu'issue des reconfigurations politiques de l'histoire coloniale après la fin de la Deuxième Guerre mondiale. Pour la réflexion, les deux revendications reposent ainsi sur des réalités culturelles qui forment encore une partie intégrante de l'actualité : pour la Nouvelle-Calédonie, il s'agit, par exemple, de la fête de l'igname, et, pour l'espace guin-ewe, et particulièrement guin de Félix Couchoro, de la fête du partage du couscous local, yèkè-yèkè. Pour les deux espaces culturels, les deux fêtes symbolisent 
l'attachement à la terre, tout comme elles actualisent les modalités internes d'organisation du réel par leurs collectivités génériques, c'est-à-dire, pour ces collectivités, la définition du réel tel que soumis au principe du calendrier mais démarqué de celui que propose la modernité et que retient l'espace issu du processus colonial. Ici, les subjectivités collectives, et les subjectivités individuelles qui s'en réclament, se positionnent, à la fois dans le temps et dans l'espace, sous le principe du calendrier annuel tel que fondé sur le souvenir et le rappel historique. On présentera brièvement la symbolique ontologique de ces deux manifestations culturelles à partir du paradigme de la géoculture, puis l'incidence de cette symbolique dans l'expression littéraire francophone de Félix Couchoro, avant de clore le propos.

\section{Les géocultures kanak et guin et leurs marqueurs}

La notion de géoculture, pour le propos, reprend en somme le courant de pensée qui cherche à faire l'état des cultures humaines dans leur capacité à transmettre la vie, critiquant en cela le modèle techniciste associé à la modernité. Dans cette perspective, géoculturelle, se comprend ainsi, par exemple, la réflexion épistémologique sur la radicalisation religieuse. La géoculture, pour ses concepteurs, Olivier Hanne et Thomas Flichy de la Neuville (2014 : 7), intègre, dans son intelligibilité, le rapport de l'individu et de sa collectivité à la mémoire historique et à sa reconfiguration en imaginaire : «L'analyse des phénomènes géopolitiques et historiques qui part des rapports identitaires et mémoriels : l'ethnicité, le temps, la religion, la perception collective, la nation et le rôle de la géographie naturelle dans son rapport aux populations. » Elle entretient également un rapport critique à la dimension économiste de la modernité : « Si le poids des facteurs économiques n'est pas négligé, il [y] est perçu comme 
dépendant d'éléments intégrateurs plus existentiels (Ibidem.). En tant que concept, la géoculture demeure, rappellent, ailleurs, ses concepteurs (Hanne et Flichy de la Neuville, 2015 : 113), «la capacité des civilisations [cultures] à perpétuer la vie». Dans son application aux cultures de référence de la géographie humaine kanak et guin-ewe, le paradigme permet de souligner le rapport foncier de telles collectivités à la perpétuation de la vie, certes, mais aussi à partir de leur milieu naturel d'existence, de même qu'en réaction à un complexe culturel associé au fait colonial, et considéré, à ce titre, comme non propice à la perpétuation de la vie. Pour la Charte du Peuple Kanak (2014 : 10), le chapitre 1 stipule, dans son alinéa 4, le lien d'ensemble de la collectivité, du Peuple kanak, à la terre :

LE LIEN A LA TERRE ${ }^{17}$ traduit la relation charnelle et spirituelle d'un clan avec l'espace naturel où se situe son tertre d'origine où apparut l'ancêtre et avec les espaces des tertres successifs qui jalonnent son histoire. Plus largement, le lien à la terre traduit la relation affective liant la famille/ le clan et la terre qui l'a vu naître et grandir.

Dans ce sens épistémologique, les fêtes de l'igname, et du couscous, ailleurs, participent respectivement de la géoculture kanak et de la géoculture guin. Pour le même chapitre 1 de la Charte, l'alinéa 13 (2014: 11) précise la valeur fondatrice de l'igname pour la collectivité, dans son ancrage dans la terre: "L'IGNAME et le TARO ${ }^{18}$ sont les symboles de la coutume kanak. Leur présence dans les cérémonies coutumières marque l'ancrage des clans dans leurs terroirs. » La Civilisation Kanak devient ainsi celle de l'igname (Ibid. : 12) :

\footnotetext{
${ }^{17}$ Dans le texte d'origine.

${ }^{18}$ Idem.
} 
Le Kanak appartient à cette terre depuis plus de 4000 ans. La Civilisation Kanak appelée aussi Civilisation de l'igname a apprivoisé d'une manière continue l'espace naturel de la montagne à la mer, au-delà de la ligne d'horizon. C'est l'Esprit de l'Ancêtre qui organise et nourrit le lien spirituel du clan et de ses membres à la Nature.

C'est à ce titre que pour l'individu kanak et sa collectivité, l'igname et sa culture, ou sa production, régulent le cycle de l'année, tout comme, chez le peuple guin, la fête du couscous, fête du partage, dans sa dénomination locale, structure le temps, celui qui est en cours et celui qui réactive la mémoire collective. Chez les Guin, la fête du couscous, fête du Nouvel An guin, est ainsi le souvenir de l'origine, de l'arrivée sur la terre étrangère, il y a longtemps, et la remémoration de l'an zéro du peuple, de son histoire dorénavant sur cette terre étrangère devenue terre d'accueil ; elle est aussi l'imprécation à l'endroit des « méchants » qui auront jalonné le cours de cette histoire (Augustin A. Amenoumve, 2001 : 489) :

Entre tristesses et joies, il est un événement primordial qui veille au fond du cœur de chaque Mina vivant chez lui ou en dehors de chez lui : c'est le yeke yeke du gbe, jour anniversaire de yèkè yèkè, fête nationale de yaka mia kin be woun e ke ou yaka e ke ou yèkè yèkè, importée d'Accra par nos ancêtres. C'est le Homon - wo Kpekple des Ghens de Ghengbo (Accra). C'est également une fête commémorative de paix, de réconciliation, enfin de malédiction à l'adresse des méchants et des sorciers.

Il s'agit du mode de régulation de l'année, avec un calendrier qui se décline à partir de la migration du Ghana voisin, devenu anglophone, vers la capitale ultérieure du « Pays Guin », Aneho, devenu, lui, francophone, par les aléas de la même histoire coloniale (Ibid. : 490) : 
En Afrique noire, les us et coutumes d'apparence simple et quelquefois grotesque, ont des fondements justifiés. Pour les faire accepter par le peuple et les asseoir définitivement, nos ancêtres ont fait des dogmes; ces dogmes deviennent des lois, les lois bien soutenues et sans failles deviennent des habitudes. [...]

Le Yêkê yêkê issu d'une institution économique, sociale et politique n'échappe pas à cette règle générale. C'est une pratique que se déroule pendant les six derniers mois environ d'une année de 13 lunes, suivant un calendrier rigoureux dont le Yêkê yêkê marque une période de 8 jours de détente.

Le plat éponyme de ce moment de l'Histoire des Guin, le Yèkè yèkè, ne se prend alors, dans l'idéal, que dans l'ambiance de fête qui caractérise le nouvel an du peuple, son renouvellement à maints autres degrés qui intègrent les rapports plus religieux ou mystiques à la terre et à sa variante de toujours, la mer, l'eau, ou l'océan ${ }^{19}$, dans les célébrations qui l'accompagnent, comme le rappelle l'historien (Ibid. : 494) :

Le samedi, c'est : memledagbe - yêkê yêkê - dougbe.

Le soleil se lève sur une ville en fête. Les danses continuent. Les ménagères affairées, chantant et dansant ont installé des doubles marmites où cuit, déjà à grand feu et à la vapeur, le yêkê yêkê. Selon les goûts et les destinations, le yêkê yêkê s'érige en obélisques blancs ou jaunes safran (couleur d'huile de palme) exposés dans des plateaux de bois, dans les cours de chaque maison, en attendant l'heure du grand déjeuner.

\footnotetext{
${ }^{19}$ Le décompte des festivités du Nouvel An guin évoque également les cérémonies vaudous guin tout comme la visite collective à la plage, pour le souvenir du peuple resté au Ghana d'origine, ou encore pour le symbolisme de la « visite » des mammifères marins, des cachalots, marque de leurs bénédictions au peuple : "Si au même moment, quelqu'un se rendait à la plage située à quelques mètres de la place d'Éla, il apercevrait un grand défilé d'énormes cachalots allant de l'ouest à l'est, ouvrant leur voie avec de gigantesques jets d'eau en arcades, arrosant et caressant leurs dos noirs et luisants avant de s'évanouir derrière eux, là-bas, dans leur majestueux sillage : Blosso wo toussi né mi. Les baleines nous bénissent » (Ibid. : 492).
} 
C'est dans l'un des derniers romans de Félix Couchoro, L'Homme à la Mercedes Benz ou Le Nouveau riche (2006; désormais HMB), que ce fait hautement identitaire, et donc fortement symbolique dans la pertinence sémiotique de l'écriture, apparaît concrètement chez l'écrivain. Là, cependant, l'évocation du yèkè-yèkè, et de son historique de faste, ne participe pas de la dynamique de l'action. Elle sert plutôt de cadre d'amplification de la propre notoriété du personnage local, quand bien même dans les conditions d'opulence que le texte problématise. Pour l'écrivain, dans la préface du roman, il s'agit, aux citoyens du nouveau pays indépendant, de pratiquer l'austérité qui garantit la souveraineté nationale :

Il ne faut pas être tellement malin pour deviner que, dans le cadre des investissements étrangers, le crédit dont jouit un pays à l'extérieur est aussi fonction de son revenu national.

«On ne prête qu'aux riches », dit un proverbe.

Paraphrasons cela, en disant : "On ne porte ses sous que chez les riches ».

Cela étant, chaque citoyen fera preuve de patriotisme en pratiquant l'austérité et en ne gaspillant point, pour des futilités, cet argent si difficilement gagné. (HMB : 581)

La mention de ce plat dans les menus riches que l'énonciateur-auteur évoque dès lors dans le roman, en le détachant de son symbolique historique immédiat, s'inscrit ainsi dans le gaspillage qu'il reproche au personnage :

Les invités prennent place à table, les dames, sur les nattes étendues à même le sol sablé.

On passe le service dans les rangs des femmes assises : platée de riz, de pâte de maïs, de Yêkè-yékê, accompagnées de caloulou de viande de bœuf, de mouton, de porc, de poulet.

Chaque invitée est à l'aise, pour s'alimenter selon ses goûts. 
Après, l'on passe d'épaisses tranches de gigot rôti, à faire descendre avec du pain. Puis, une tournée de vin rouge pur vient arroser ces riches nourritures. Enfin, une distribution de gâteau-cake et de biscuits fins, sans oublier les crêpes-atsonmo.

À la table des hommes, sandwiches divers, bœuf bouilli aux pommes de terre, salade verte, poulets et dindes en rôts garnis, Yêkèyékê également, gâteaux et biscuits, vin rouge et bière glacée, au choix. (HMB : 612-613)

Dans cette problématisation, qui révèle la prise de position axiologique du narrateur, le personnage devenu riche et imprudent dans la gestion de cette richesse dont il hérite de son père, aura toutefois eu des problèmes du fait aussi de la «conspiration» de monde des finances expatrié et propriétaire des institutions financières dans le pays pourtant indépendant. Le texte sauvera in extremis le personnage de la faillite par le deus ex machina d'un billet de la loterie nationale que le futur gendre du personnage achète et qui lui fait gagner le gros lot. La célébration de l'intervention bénéfique du pays indépendant et venant à la rescousse indirecte du personnage en bute aux réminiscences de l'histoire coloniale, réinsère la célébration de la fête du couscous traditionnel dans son symbolisme identitaire et anticolonialiste, à la rigueur. Il en va ainsi de la scène finale, présentée dans l'épilogue du roman, où le personnage se redresse face au banquier étranger, coupable de « conspiration » contre le « fils du pays » :

Le banquier fit apporter deux coupes et une bouteille de champagne.

- Le Togo, votre pays, fête aujourd'hui son Independance Day. Profitons donc de cette entrevue heureuse, pour avaler un peu de mousseux en ce début de festivité nationale.

L'instant d'après, Richard prenait congé, l'esprit bourré de réflexions.

Berger s'accrochait encore à ses « ordres de Paris », alors que le gros œuvre du méchant complot avait été bien cuisiné « on the spot», sur la place de Lomé, avec préavis pour Paris. 
Richard devina que, lui parti, le banquier allait sauter sur son téléphone pour sonner le cessez-le-feu chez chaque membre de la ligue occulte de «mécontents », ceux-là qui voulaient la mort du pécheur, et non sa conversion. (HMB : 640)

À travers le plat, personnage non-anthropomorphe et secondaire dans l'intrigue, la fête des Guin qu'il réactivait prend toute sa valeur dans la permanence qu'elle accorde à l'individu, tant du point de vue ontologique que du point de vue politique dans le roman. À travers elle, c'est aussi le retour à la «Terre des Aïeux », chez l'écrivain, dans son caractère immémorial. L'expression constitue également le titre de l'hymne national de l'État postcolonial sorti de 1'histoire coloniale. J. Améla (2001 : 442) le rappelle dans la certitude de l'individu-sujet Guin quant à la prévalence de sa culture, de la valeur de l'ancestralité donc, face à l'histoire coloniale : «Ce choix résolument optimiste est manifesté dans le slogan "mianto miano miade, nous habitons sur la terre de nos coutumes, de nos ä̈eux", repris dans les rythmes du tam-tam comme dans les chansons, et même dans l'hymne national togolais, Terre de nos aïeux. »

Pour la tradition culturelle kanak, la culture de l'igname, qui régule le cycle de l'année, trouve également son point culminant dans la fête qui marque la saison des récoltes, ou de l'igname nouveau. Son intérêt, par-delà le rappel de l'ordre de la nature dans l'existence de l'individu et de la collectivité, réside, là aussi, dans le moment de rencontre qu'elle occasionne. Ce moment associe à la célébration de ce lien avec la terre celui qui existe entre les individus et les collectivités, clans et familles, et forme la base de l'organisation sociale $\mathrm{du}$ peuple kanak: «Le clan et ses composantes internes (sous clans, branches, maisons) est la base de l'organisation sociale Kanak », dit encore la Charte $d u$ 
Peuple Kanak (2014 : 15). Pour l'écrivaine et militante kanak de longue date, Déwé Gorodé, la culture de l'igname est ce lien indéfectible du peuple kanak à la terre, dans le sens où ce rapport à la terre définit le réel ontologique pour le peuple. Dans le commentaire qu'il présente d'un des recueils de nouvelles de l'auteure, Par les Temps qui courent, Hamid Mokaddem (1998: 99) rappelle combien le cycle de la culture de l'igname constitue une véritable horloge du temps kanak, et apparaît de la sorte chez Déwé Gorodé :

La terre habite le Kanak. La terre rythmée par le cycle de la culture de l'igname, véritable horloge du temps kanak. Déwé Gorodé sait bien ce que signifie ce lien substantiel, nourricier de la terre. Le lieu des vivants, celui des morts, des esprits et des ancêtres, des endroits sacrés, tabous, le lieu des tertres où s'originent les généalogies des clans. La terre, où coexistent les paradis et les tribus, Déwé la célèbre dans la langue qui l'a nourrie, la langue maternelle, le paicî [...].

Ce rapport fondamental permet ensuite à l'écriture littéraire d'établir le lien entre la coutume, la mémoire, la tradition et tout le tort que l'histoire coloniale aura introduit dans le réel du peuple. La terre qu'évoque ici l'individu écrivant est celle-là qui porte désormais les stigmates de l'histoire coloniale qui se poursuit (Ibid. : 100) :

La terre demeure malgré l'artifice colonial. Ce « séjour paisible» qu'évoque le poète est perturbé par les propriétés foncières et extensives des colons, le business, le capitalisme. Dans les tribus de la chaîne centrale de la côte Est, la terre réconforte l'homme qui vit en paix. L'homme, proche d'endroits sacrés, ne hausse pas la voix, de peur de réveiller les esprits. La Nature parle, produit des signes, exprime des changements bienfaisants ou inquiétants, des repères spatiaux, temporels, qui manifestent les apparitions, les formes des esprits. La terre exprime des histoires. Dans les pays kanak, on appelle les gens par leur lieu d'habitation. C'est une marque de respect. 
Ce même rapport à la terre définit les Guin, peuple migrant et pourtant fondateur du pays post-colonial, le Togo. À travers la fête du couscous local, il institue un calendrier qui excède le temps colonial et constitue à chacune de ses célébrations un rappel de la contingence de l'histoire coloniale.

Pour le peuple guin, ou mina, dont la migration d'Accra, au Ghana, à l'actuel sud-est du Togo date en effet de la fin du XVII ${ }^{\mathrm{e}}$ siècle, la cérémonie du nouvel An sert autant de mémoire historique que de rassemblement des fils et filles du «Pays ». C'est au cœur de cette célébration que se situe la fête du partage, yèkè-yèkè, au sens littéral de "Nous l'avons partagé librement et gratuitement ». La légende dit qu'elle aurait été occasionnée bien avant la migration du $17^{\mathrm{e}}$ siècle par une famine de laquelle le peuple guin se serait sorti par le maïs qui avait fini par pousser pour le nourrir et le sauver de la mort ; l'historien en donne l'une de ses versions (Amenounve, op. cit. : 490) :

Il y eut la guerre entre les Ga et un autre peuple, probablement les Akwamu. Les ennemis encerclèrent les champs des $\mathrm{Ga}$ et les empêchèrent d'aller récolter; ils commencèrent à mourir de faim jusqu'à ce qu'une nuit, pendant que les ennemis étaient tous endormis, ils se jetèrent sur eux et les exterminèrent. Du Kpo Kpoï, genre de couscous et une sauce au jus de noix de palme et du poisson furent préparés. Après le festin, trépignant tous de joie, ils disaient «Awoo, awoo, yi awoo», louanges aux divinités protectrices de la tribu; ce qui signifie : «adieu la faim et soient à jamais éloignés la famine et la disette ».

Une autre version la situe dans la migration initiale du Nigéria vers le Ghana, où le couscous aura également sauvé le peuple de la famine... (Ibid. : 489-490) Pour les Guin, le yèkè yèkè, au-delà du plat historique, est aussi une fête qui trouve son 
symbolisme dans l'histoire politique du Togo, où, par les aléas de l'histoire coloniale, les «méchants » et les « sorciers », dans le compte rendu qu'en fait l'historien (Ibid. : 489), ce peuple fondateur se sera trouvé mis à l'écart de la gestion politique de l'entité étatique ayant émergé de l'histoire coloniale. C'est de ce cadre politique et historique à la fois que les Guin réinstituent le rapport à la terre qui, pour eux, demeure indéfectible, même devant une histoire coloniale qui infirme la précellence de la terre des aïeux. C'est de même au nom de cet attachement à l'histoire des ancêtres et de la terre, celle d'origine et celle de l'établissement ultérieur du peuple, que naîtra le mouvement politique de l'ère coloniale où se trouve fédérée la terre des Ewe ayant accueilli les migrants guin d'antan. Ce mouvement politique demandera d'abord la réunification de l'ancien territoire des Guin-Ewe devenu colonie allemande puis confié à la France et à la Grande-Bretagne après la Première Guerre mondiale. Face aux conditions particulièrement antagoniques de la tutelle française, le mouvement nationaliste ewe se résoudra à demander l'indépendance d'une portion de la terre des aïeux, laquelle portion constitue le territoire du Togo post-colonial. Toute l'œuvre littéraire et politique de Félix Couchoro participera de ce mouvement nationaliste ewe, où il s'agit, pour l'écrivain, telle Déwé Gorodé en Nouvelle-Calédonie, d'en appeler à une résistance fondée sur l'appel à un retour à la terre et opposée à l'idéologie coloniale dans son ensemble.

\section{Conclusion}

Le discours sur la terre prend ainsi diverses formes dans les deux exemples évoqués de la Nouvelle-Calédonie et du pays Guin chez les deux écrivains. Pour la réflexion épistémologique qui s'intéresse à ces deux cas, la terre constitue une constante ontologique à laquelle se réfèrent les individus pour apposer un 
discours sur le réel, celui de référence de leurs écritures, et pour infirmer ceux des aspects de la réalité préjudiciables à l'intégrité de ce réel. En cela, leur discours sur la terre devient multiforme car s'en prenant à une histoire proprement francophone, celle de la colonisation française, pour en dénoncer les divers mécanismes d'implantation et de perpétuation. Si Déwé Gorodé ajoute la langue paicî qu'elle parle et enseigne, étant sa langue d'origine, à son écriture en français, Félix Couchoro en fait de même avec toutes les langues du pays GuinEwe par lesquelles s'infirme ainsi l'hégémonie instituée de la langue française. Pour la même réflexion, suivre le discours de l'individu écrivant francophone sur sa terre, son territoire, son pays, devient la mesure du positionnement ontologique qui permet de redéfinir le fait francophone dont il participe, et, partant, le fait francophone littéraire lui-même, d'un espace local spécifique à un autre.

Dans le projet d'indépendance de la Nouvelle-Calédonie face aux conditions particulièrement antagonistes de l'histoire coloniale, la permanence du complexe géoculturel évoqué dans la réflexion permet de repenser l'identité « francophone » à venir du peuple kanak. Le projet indépendantiste, tel celui qui maintient le souvenir de la terre d'origine chez les Guin, malgré les conditions politiques de l'histoire post-coloniale, demeure ancré dans cette permanence que convoque la fête de l'igname devenue marqueur identitaire et paradigme ontologique, tout à la fois. Elle fait augurer de ce moment où le retour de l'histoire à la terre, pour le peuple, signifierait également cette accession à l'indépendance que le peuple aura demandé avec colère et résignation parfois face aux «méchants» et aux «sorciers» qu'évoque l'historien guin. Si la Charte du Peuple Kanak envisage ce moment de la fin de la domination coloniale, il reste que c'est sur la base du complexe géoculturel autochtone qu'il faudra penser alors la reconfiguration de l'espace collectif ayant émergé de l'indépendance. Les 
pièges de la modernité devraient permettre d'évoquer une telle nécessité susceptible de garantir la pérennité de la collectivité sur ses terres ancestrales et dans les conditions historiquement favorables à son intégrité holistique et à celles des collectivités qui se sont retrouvées avec elle sur ces terres ancestrales. L'échec de la modernité dans ses crises de la fin du $\mathrm{XX}^{\mathrm{e}}$ siècle et des débuts du $\mathrm{XIX}^{\mathrm{e}}$ siècle permettent de penser ce rapport nécessaire à la terre dans l'organisation de la collectivité. De penser les identités francophones à partir de leurs subjectivités constitutives ouvre ainsi la voie à cette pensée de l'aprèsmodernité que l'écrivain francophone intègre dans l'expression de sa subjectivité francophone. 


\section{Bibliographie}

Amela, Janvier, « Aneho, espace littéraire dans l'œuvre de Couchoro », dans N. L. Gayibor, Le Tricentenaire d'Aneho et du pays Guin. Volume 2 : Société, culture et développement en pays Guin, Lomé, Presses de l'Université du Bénin, 2001, p. 441-455.

Amenoumve, A. Augustin, «Le yaka mia kin ou le nouvel an guin », dans N. L. Gayibor, Le Tricentenaire d'Aneho et du pays Guin. Volume 2 : Société, culture et développement en pays Guin, Lomé, Presses de l'Université du Bénin, 2001, p. 489-497.

Assiom, M. K., et Eccoe-Aduadje, F. F., «Culture et société en pays guin », dans N. L. Gayibor, Le Tricentenaire d'Aneho et du pays Guin. Volume 2 : Société, culture et développement en pays Guin, Lomé, Presses de l'Université du Bénin, 2001, p. 469-472.

Couchoro, Félix, L'Homme à la Mercedes Benz ou Le Nouveau riche [1968], Euvres complètes, Tome 2, London, Ontario, Mestengo Press, 2006, p. 579-641.

Dauphiné, Joël, « La Nouvelle-Calédonie de 1945 à nos jours », Notre Librairie, n 134 , mai-août 1998, p. 18-26.

Hanne, Olivier et Flichy de la Neuville, Thomas (dir.), Guerres à l'horizon, Panazol, Éditions Lavauzelle Graphic, 2014.

Hanne, Olivier et Flichy de la Neuville, Thomas, Géoculture, plaidoyer pour une civilisation durable, Panazol, Éditions Lavauzelle Graphic, 2015.

Lawson-Hellu, Laté, «Le discours sur la terre et la configuration épistémologique du texte francophone », Les Cahiers du GRELCEF, $\mathrm{n}^{\circ}$ 10, 2018, p. 11-28.

Mokaddem, Hamid, «Par les temps qui courent de Déwé Gorodé ou l'exigence de la pensée dans la parole kanak », Notre Librairie, n 134 , mai-août 1998, p. 95-102 
Sénat Coutumier de la Nouvelle-Calédonie, Charte du Peuple Kanak. Socle Commun des Valeurs et Principes Fondamentaux de la Civilisation Kanak, Kowe-Kara, Nouvelle-Calédonie, avril 2014. [https://www.legitimus.ca/static/uploaded/Files/Documents/Charte-Kanak2014.pdf]. 